\title{
Lentiviral delivery of CTLA-4 shRNA improves the expansion of cytokine-induced killer cells and enhances cytotoxic activity in vitro
}

\author{
TAO RUI ${ }^{1}$, XIANGDONG CHENG $^{1}$, HAO WU $^{2}$, FUWEI WANG $^{2}$, ZAIYUAN YE $^{3}$ and GUOQING WU ${ }^{2}$ \\ ${ }^{1}$ Department of Gastroenterology, Zhejiang Provincial Hospital of Traditional Chinese Medicine, \\ Hangzhou, Zhejiang 310000; Departments of ${ }^{2}$ Oncology and ${ }^{3}$ Gastroenterology, \\ Zhejiang Provincial People's Hospital, Hangzhou, Zhejiang 310014, P.R. China
}

Received November 13, 2015; Accepted July 7, 2017

DOI: $10.3892 / \mathrm{ol} .2017 .7376$

\begin{abstract}
Cytokine-induced killer (CIK) cells are in vitro-expanded cells harboring potent toxicity against tumor cells. Recently, it was identified that the cytotoxicity and proliferation of CIK cells are restricted by a prolonged CIK cell culture period. Cytotoxic T lymphocyte-associated antigen 4 (CTLA-4) serves a negative role in $\mathrm{T}$ cell activation and proliferation. This study aims to determine whether CTLA-4 expression is associated with the inhibition of CIK cells. CIK cells were generated from peripheral blood mononuclear cells (PBMCs), and CTLA-4 shRNA (shCTLA-4) lentivirus was applied to knockdown CTLA-4 expression in CIK cells. The proliferation of CIK cells was evaluated following shCTLA-4 lentiviral transduction, and the cytotoxicity of CIK cells was investigated using the CytoTox 96 Non-Radioactive Cytotoxicity assay. The expression of CTLA-4 in CIK cells was significantly increased, compared with that in PBMCs. The shCTLA-4 lentivirus efficiently knocked down the expression of CTLA-4 in CIK cells. The shCTLA-4 lentivirus transduction of CIK cells promoted the proliferation of CIK cells in vitro (3.18 \pm 0.19 -fold vs. 2.42 \pm 0.29 -fold). Furthermore, the cytotoxicity of shCTLA-4 lentivirus-transduced CIK cells was significantly improved when compared with that of control shRNA lentivirus-transduced CIK cells $(54.5 \pm 2.13 \%$ vs. $30.5 \pm 1.67 \%$ ). Thus, the suppression of CTLA-4 expression increases cytotoxicity and ex vivo expansion of CIK cells, which indicates a clinical significance for CTLA-4 blockade in CIK cell therapy.
\end{abstract}

Correspondence to: Dr Guoqing Wu, Department of Oncology, Zhejiang Provincial People's Hospital, 158 Shangtang Road, Hangzhou, Zhejiang 310014, P.R. China

E-mail: gqwuzsu@hotmail.com

Key words: proliferation, CTLA-4, cytokine-induced killer cell, cytotoxicity

\section{Introduction}

Cancer immunotherapy exerts beneficial effects through mediating tumor cell regression (1), which relies on activating antitumor $\mathrm{T}$ cells. Co-stimulatory molecules, such as CD80 (B7-1) and CD86 (B7-2), expressed on the surface of dendritic cells (DCs) or other antigen presenting cells (APCs), regulate $\mathrm{T}$ cell activation and proliferation by interacting with CD28 $(2,3)$. Conversely, upon activation, cytotoxic T lymphocyte-associated antigen 4 (CTLA-4) is expressed on the T cell surface, and also interacts with CD80 and CD86. However, contrary to CD28, CTLA-4 serves an inhibitory co-stimulatory role in regulating $\mathrm{T}$ cell activation and proliferation $(4,5)$. It was reported that CTLA-4 downregulates the activation of T cells by inhibiting IL-2 production and cell cycle progression (6). It has been verified that CTLA-4 competes with CD28 and acts as an inhibitory receptor to influence T cell function (7).

Adoptive cell therapy (ACT) is a promising anticancer therapy option (8). Cytokine-induced killer (CIK) cells, a heterogeneous subset of expanded lymphocytes, exhibit notable cytotoxic activity against tumor cells (9-11). CIK cells are generated from peripheral blood mononuclear cells (PBMCs), with a cytokine cocktail including interferon (IFN)- $\gamma$, anti-CD3 monoclonal antibody, interleukin (IL)-2 and IL-1 $\alpha$ (12). Clinically, a limited expansion rate and low in vivo toxicity are significant shortcomings of CIK cell therapy. The present study hypothesized that CTLA-4 expression increases when PBMCs are induced into CIK cells during in vitro culture. In the present study, lentivirus-mediated RNA interference was employed to knock down CTLA-4 expression in CIK cells and explore the effects of CTLA-4 inhibition on the proliferation and toxicity of CIK cells.

\section{Materials and methods}

Cell culture. The A549 human lung carcinoma cell line (no. 59239596; American Type Culture Collection, Manassas, VA, USA) was cultured in RPMI-1640 medium (Thermo Fisher Scientific, Inc., Waltham, MA, USA) supplemented with $10 \%$ fetal bovine serum (FBS; Thermo Fisher Scientific, Inc.). PBMCs from healthy adults were collected by cell 
apheresis (Fresenius Kabi Asia-Pacific, Ltd., Wanchai, China). CIK cells were generated by culturing PBMCs in RPMI-1640 supplemented with $10 \%$ FBS and containing $1,000 \mathrm{IU} / \mathrm{ml}$ recombinant IFN- $\gamma$ (Shanghai Chemo Wanbang Biopharma Co., Ltd., Shanghai, China). At 24 h, $100 \mathrm{ng} / \mathrm{ml}$ anti-CD3 antibody (Wuhan Institute of Biological Products Co., Ltd., Wuhan, China), 1,000 IU/ml IL-2 (Four Rings Biotechnology, Beijing, China) and $1 \mathrm{ng} / \mathrm{ml}$ IL-1 $\alpha$ (Invitrogen; Thermo Fisher Scientific, Inc.) were added. From day 5, the cells were replenished every 3 days with fresh medium containing $1,000 \mathrm{IU} / \mathrm{ml}$ IL-2. All cells were culturedat $37^{\circ} \mathrm{Cin} 5 \% \mathrm{CO}_{2}$.

Cell transduction of CTLA-4 shRNA (shCTLA-4) lentiviral particles. shCTLA-4 lentiviral particles (Hanbio Biotechnology Co., Ltd., Shanghai, China), containing a 29-mer shRNA sequence 5'-GGAATGAGTTGACCTTCC TAGATGA-3', were used to knockdown the expression of CTLA-4 in CIK cells. On day 10, CIK cells were transduced with shCTLA-4 lentiviral particles at multiplicity of infection of 10. A total of $96 \mathrm{~h}$ later, the transduction efficiency was estimated by detecting CIK cells expressing green fluorescence protein under a fluorescence microscope.

Polymerase chain reaction (PCR). Total RNA was isolated using an RNeasy Mini kit (Qiagen GmbH, Hilden, Germany), and reverse transcribed as single-stranded cDNA. cDNA was then used as the template to amplify CTLA- 4 via PCR with the following primers: Forward, 5'-GACCTGGCCCTGCACTCT CCTGTTT-3' and reverse, 5'-ACTGTCACCCGGACCTCA GTGGCTT-3'. GAPDH was employed as the internal control. Primers for the amplification of GAPDH are as follows: Forward, 5'-TGCCTCCTGCACCACCAACT-3' and reverse, 5'-CCCGTTCAGCTCAGGGATGA-3'. PCR was carried out at $94^{\circ} \mathrm{C}$ for $30 \mathrm{sec}, 55^{\circ} \mathrm{C}$ for $30 \mathrm{sec}$ and then $72^{\circ} \mathrm{C}$ for $30 \mathrm{sec}$.

Western blotting. For protein analysis, total protein was extracted from $1 \times 10^{7}$ CIK cells using RIPA lysis buffer (BestBio, Shanghai, China) and then quantified using a BCA kit (Pierce; Thermo Fisher Scientific, Inc.). Equal amount of whole cell lysates (20 $\mu \mathrm{g}$ per lane) were separated using SDS-PAGE on a $10 \%$ gel and transferred to a polyvinylidene difluoride membrane (Bio-Rad Laboratories, Inc., Hercules, CA, USA). The membrane was blocked using $2.5 \%$ non-fat milk for $1 \mathrm{~h}$ at room temperature and then incubated with rabbit monoclonal antibody against human CTLA-4 (cat. no. SC-9094; 1:200; Santa Cruz Biotechnology, Inc., Dallas, TX, USA) or rabbit polyclonal antibody against human GAPDH (cat. no. SC-25778; 1:1,000; Santa Cruz Biotechnology, Inc.) for $2 \mathrm{~h}$ at $25^{\circ} \mathrm{C}$ followed by incubation with a horseradish peroxidase-conjugated goat anti-rabbit immunoglobulin $\mathrm{G}$ antibody (cat. no. SC-2004; 1:2,000; Santa Cruz Biotechnology, Inc.) for $1 \mathrm{~h}$ at $25^{\circ} \mathrm{C}$ prior to detection with chemiluminescence (FluorChem $^{\mathrm{TM}}$ HD2 system; ProteinSimple; Bio-Techne, Minneapolis, MN, USA). The expression of CTLA-4 was normalized to that of GAPDH.

Cytotoxicity assay. CIK cell-mediated cytotoxicity was assessed using the CytoTox $96^{\circledR}$ Non-Radioactive Cytotoxicity Assay (Promega Corporation, Madison, WI, USA), according to the manufacturer's protocol. Briefly, shCTLA-4 lentiviral particle-transduced CIK cells, control shRNA (shControl) lentiviral particle-transduced CIK cells and the CIK cells without lentivirus transduction were suspended in RPMI-1640 medium, supplemented with 5\% FBS, at a density of $2 \times 10^{6}$ cells $/ \mathrm{ml}$. The control and experimental wells were set up using a round-bottom 96-well culture plate. The wells which only contained CIK cells served as the control for the spontaneous LDH release effector cells; the experimental wells contained CIK cells and A549 cells at a ratio of 20:1. Cells were centrifuged at $250 \mathrm{x} \mathrm{g}$ for $4 \mathrm{~min}$ at $20^{\circ} \mathrm{C}$ after incubation at $37^{\circ} \mathrm{C}$ for $4 \mathrm{~h}$. For target cell maximum LDH release control wells, the lysis solution was added $45 \mathrm{~min}$ prior to supernatant harvest. A total of $50 \mu l$ supernatant from each well of the assay plate was transferred to a flat-bottom 96-well plate that was pre-loaded with $50 \mu \mathrm{l} /$ well reconstituted substrate mix. Following incubation at room temperature while protected from light for $30 \mathrm{~min}, 50 \mu \mathrm{l}$ stop solution was added to each well followed by reading the absorbance at $490 \mathrm{~nm}$. The cytotoxicity of CIK cells was calculated as follows: [(Experimental-effector spontaneous-target spontaneous)/(target maximal-target spontaneous)]x 100 .

To further confirm the cytotoxicity of CIK cells, a more convenient and intuitive cell counting assay was used to detect the cytotoxicity of the shCTLA-4 lentiviral particle-transduced CIK cells. As aforementioned, the CIK cells transduced with shCTLA-4 lentiviral particles or shControl lentiviral particles, and the CIK cellswithout lentiviral transduction were co-cultured with A549 cells at a ratio of 20:1 (effector cells:target cells). For the negative group, only A549 cells were seeded and cultured for $24 \mathrm{~h}$. According to the morphology of adhesive A549 cells and suspended CIK cells, the residual A549 cells from all groups were observed under a microscope and counted following trypan blue dye exclusion. The cytotoxicity of effector cells could be deduced from the numeration of A549 cells that survived the CIK cells.

Statistical analysis. All statistical analyses were performed using SPSS software (version 19.0; IBM Corp, Armonk, NY, USA). The band signals were analyzed using Quantity One software (version 4.6.2; Bio-Rad Laboratories, Inc.). The data are presented as the mean \pm standard error of the mean. Two or multiple group comparisons were performed using a Student's t-test or one-way analysis of variance with Fisher's least significant difference post hoc test, respectively. $\mathrm{P}<0.05$ was considered to indicate a statistically significant difference.

\section{Results}

The expression of CTLA-4 increases with the induction of CIK cells. To compare the expression levels of CTLA-4 between CIK cells and the corresponding PBMCs, CIK cells and PBMCs were used to prepare total RNA and protein. As evaluated using RT-PCR and western blot analysis, the mRNA and protein expression levels were much higher in CIK cells than those in PBMCs (Fig. 1A and B). These observations suggested that CTLA-4 expression increases during the induction of CIK cells.

shCTLA-4 lentivirus efficienctly inhibits CTLA-4 expression. The shCTLA-4 lentiviral particles were employed to knock 
A

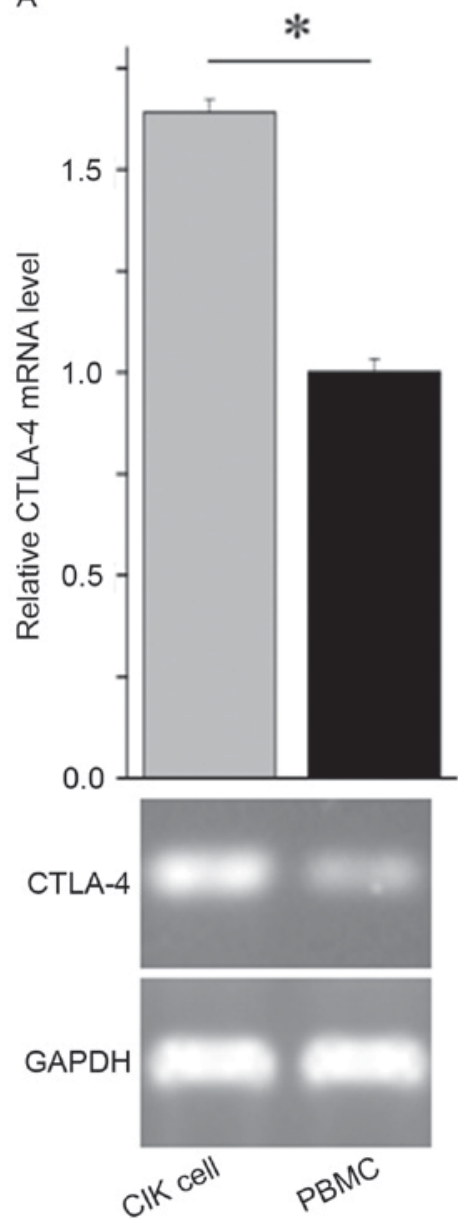

B
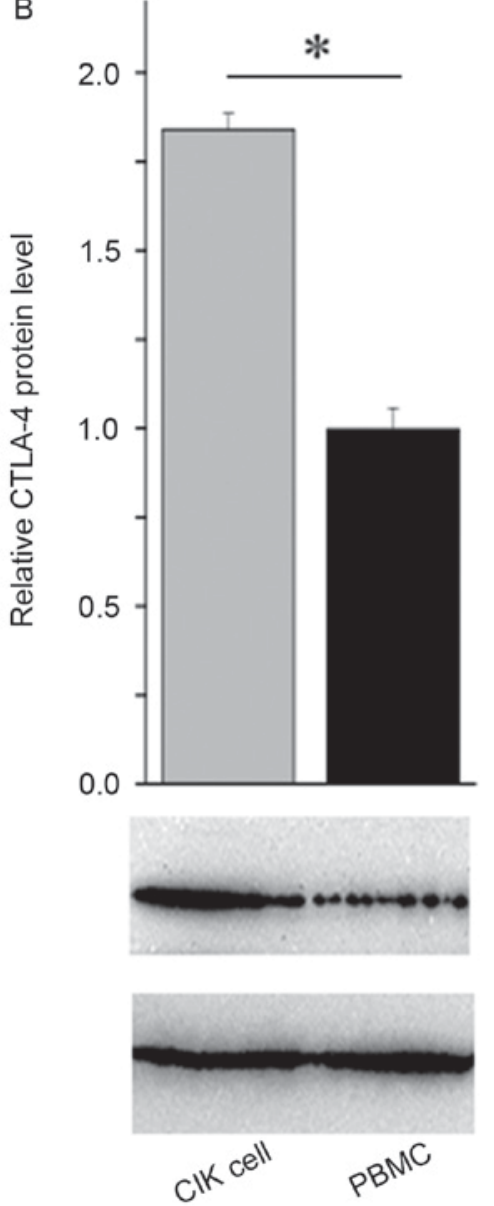

Figure 1. CTLA-4 expression was higher in CIK cells than in PBMCs. (A) The CTLA-4 mRNA expression in PBMC or CIK cells. ${ }^{*} \mathrm{P}<0.05$. Top panel, qPCR analysis of CLTA-4 mRNA expression in PBMCs and CIK cells. CTLA-4 mRNA expression levels were normalized to GAPDH. CLTA-4 mRNA expression level in CIK cells was normalized to 1 for PBMCs. Bottom panel, agarose gel images indicating the mRNA expression of CTLA-4 and GAPDH in PBMCs and CIK cells. (B) The CTLA-4 protein expression in PBMC or CIK cells. " $\mathrm{P}<0.01$. Top panel, western blot analysis of CLTA-4 protein expression in PBMCs and CIK cells. CTLA-4 protein expression levels were normalized to GAPDH. CLTA-4 protein expression level in CIK cells was normalized to 1 for PBMCs. Bottom panel, representative images indicating the protein expression of CTLA-4 and GAPDH in PBMCs and CIK cells. All the data presented are mean values from three independent experiments and plotted as the mean \pm standard error of the mean. CTLA-4, cytotoxic T lymphocyte-associated antigen 4; CIK, cytokine-induced killer; PMBC, peripheral blood mononuclear cells; qPCR, quantitative polymerase chain reaction.

down CTLA-4 expression in CIK cells. As determined by PCR, shCTLA-4 lentiviral particles significantly decreased CTLA-4 mRNA levels in CIK cells when compared with the shControl lentiviral particles (Fig. 2A). As analyzed by western blotting, shCTLA-4 lentiviral particles significantly reduced the expression levels of CTLA-4 protein in CIK cells when compared with the shControl lentiviral particles (Fig. 2B). These data indicate that shCTLA-4 lentiviral particles can successfully silence the expression of CTLA-4 in CIK cells.

Silencing CTLA-4 promotes the proliferation of CIK cells. To examine whether silencing CTLA-4 with shCTLA-4 lentivirus affects the proliferation rate of CIK cells, CIK cells were transduced with shCTLA-4 lentivirus or shControl lentivirus. A total of $96 \mathrm{~h}$ after viral transduction, cell counting was performed. Morphologically, the shCTLA-4 lentivirus-transduced CIK cells presented with more agglomeration than the shControl lentivirus-transduced CIK cells did (Fig. 3A). Compared with control transduction of shControl lentivirus, the transduction of shCTLA-4 lentivirus promoted the ex vivo expansion of CIK cells (Fig. 3B). These findings indicated that transduction of shCTLA-4 lentivirus enhances the proliferation of CIK cells.

Silencing CTLA-4 improves the cytotoxicity of CIK cells. As determined by a CytoTox 96 Non-Radioactive Cytotoxicity Assay, the shCTLA-4 lentivirus-transduced CIK cells caused more LDH release against A549 cells compared with the shControl lentivirus-transduced CIK cells did (Fig. 4A). A further cell counting assay indicated that, compared with the shControl lentivirus-transduced CIK cells, a decreased number of A549 cells survived under the cytotoxic activity of shCTLA-4 lentivirus-transduced CIK cells (Fig. 4B and C). These findings indicate that the cytotoxicity of CIK cells against A549 cells can be enhanced through the inhibition of CTLA-4 expression in CIK cells.

\section{Discussion}

As a potential anti-tumor ACT, CIK cells have been extensively tested in clinical practice (13-16). The ex vivo expansion rate and cytotoxicity are two key factors that determine the therapeutic 
A
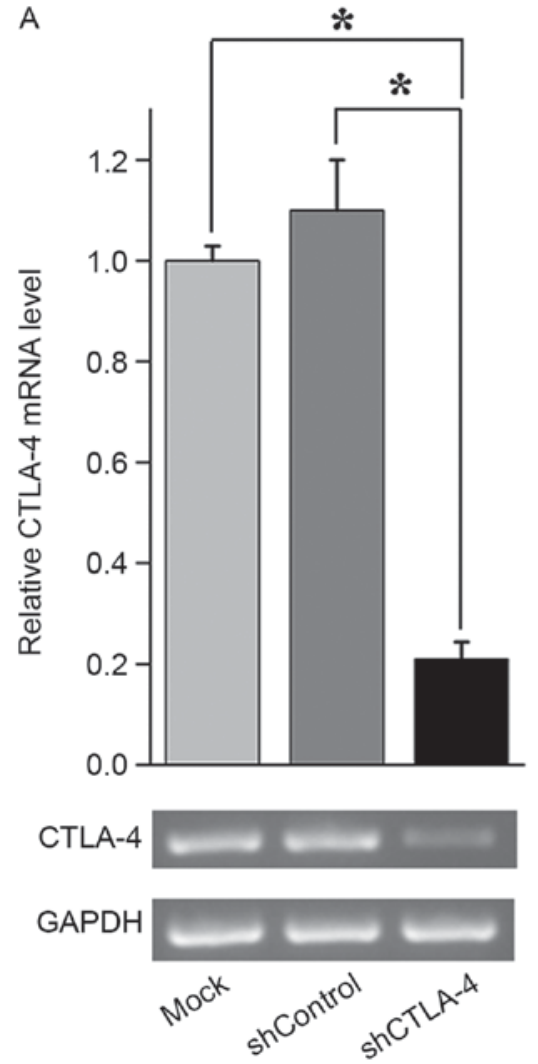

B

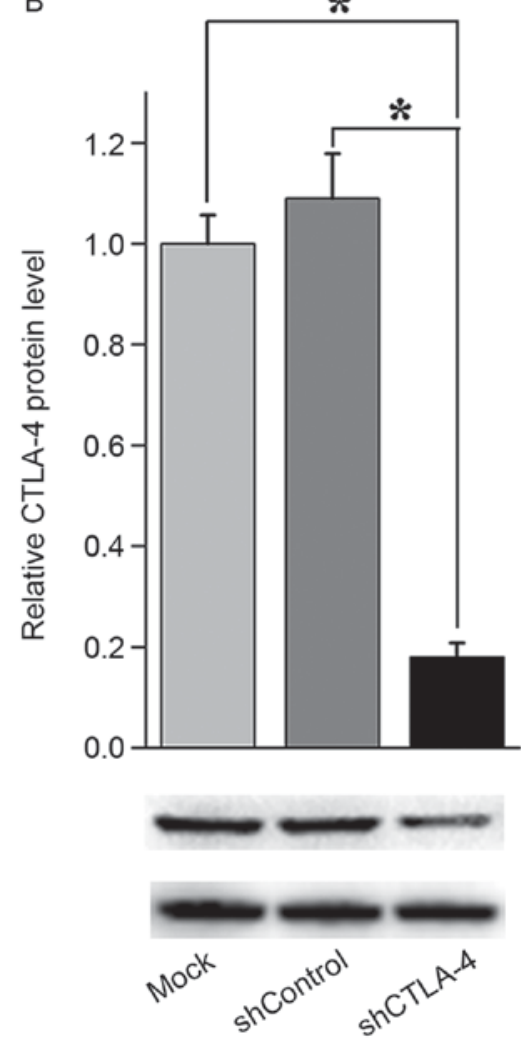

Figure 2. CTLA-4 shRNA knocked down CTLA-4 expression in CIK cells. (A) Agarose gel images and qPCR analysis indicated that CTLA-4 mRNA expression was down-regulated in shCTLA-4 lentiviral particles transduced CIK cells compared with mock transduced CIK cells or shControl lentiviral particles transduced CIK cells. * $\mathrm{P}<0.01$. CTLA-4 mRNA expression levels were normalized to GAPDH. CTLA-4 mRNA expression from shControl lentiviral particles transduced CIK cells and shCTLA-4 lentiviral particle-transduced CIK cells were normalized to 1 for mock transduced CIK cells. (B) Western blot analysis indicated that CTLA-4 protein expression was down-regulated in shCTLA-4 lentiviral particles transduced CIK cells compared with mock transduced CIK cells or shControl lentiviral particles transduced CIK cells. ${ }^{*} \mathrm{P}<0.01$. CTLA-4 protein expression levels were normalized to GAPDH. CTLA-4 protein expression from shControl lentiviral particles transduced CIK cells and shCTLA-4 lentiviral particles transduced CIK cells were normalized to 1 for mock transduced CIK cells. The data presented are mean values from three independent experiments and plotted as the mean \pm standard error of the mean. CTLA-4, cytotoxic T lymphocyte-associated antigen 4; CIK, cytokine-induced killer; PMBC, peripheral blood mononuclear cells; qPCR, quantitative polymerase chain reaction; sh, short hairpin.

A

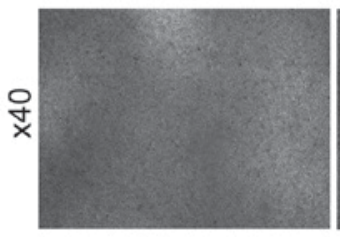

Mock

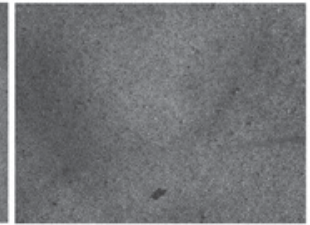

shControl

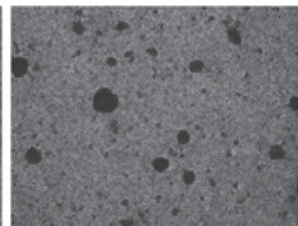

ShCTLA-4

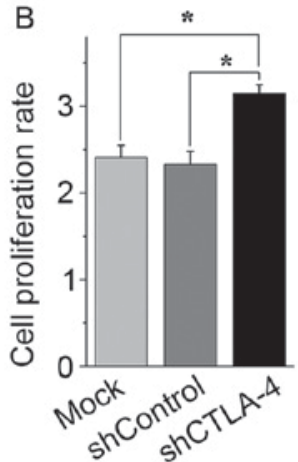

Figure 3. Inhibition of CTLA-4 expression promoted CIK cell expansion in vitro. CIK cells on day 10 were transduced with shCTLA-4 lentiviral particles, shControl lentiviral particles, or mock transduced. A total of $96 \mathrm{~h}$ after cell transduction, CIK cells were harvested and counted to evaluate cell expansion efficiency. (A) CIK cells mock transduced (left panel), transduced with shControl lentiviral particles (middle panel), or transduced with shCTLA-4 lentiviral particles (right panel). Magnification, x40. (B) Compared with mock transduced CIK cells or shControl lentiviral particles transduced CIK cells, shCTLA-4 lentiviral particles transduced CIK cells exhibited a higher expansion rate. ${ }^{*} \mathrm{P}<0.01$. Each experiment was performed three times. The data are plotted as the mean \pm standard error of the mean. CTLA-4, cytotoxic T lymphocyte-associated antigen 4; CIK, cytokine-induced killer; PMBC, peripheral blood mononuclear cells; qPCR, quantitative polymerase chain reaction; sh, short hairpin.

efficacy of CIK cells. CIK cells specifically kill tumor cells via the secretion of IFN- $\gamma$, tumor necrosis factor- $\alpha$ or perforin (16). However, the activation of immune suppression pathways induces T cell anergy (17). By competing with CD28, CTLA-4 serves anegative role in the regulationof T-cell activation. CTLA-4 prohibits T cell activation through the inhibition of signal transduction of the $\mathrm{T}$ cell receptor and transmitting the inhibitory signal to T cells (17-19). These maybe associated with 
A

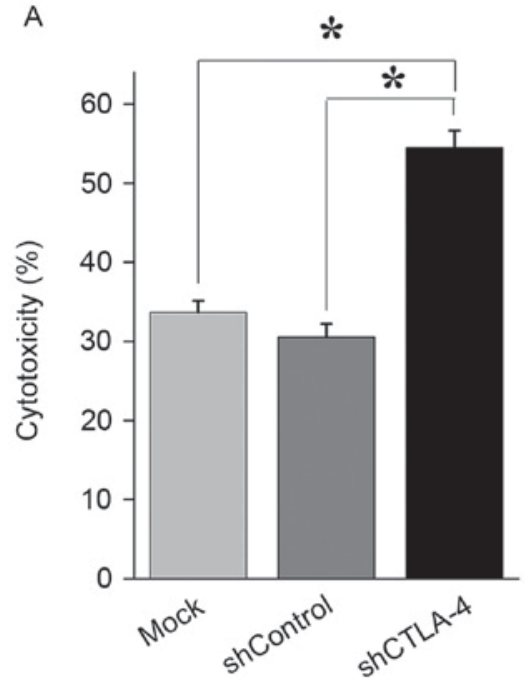

B

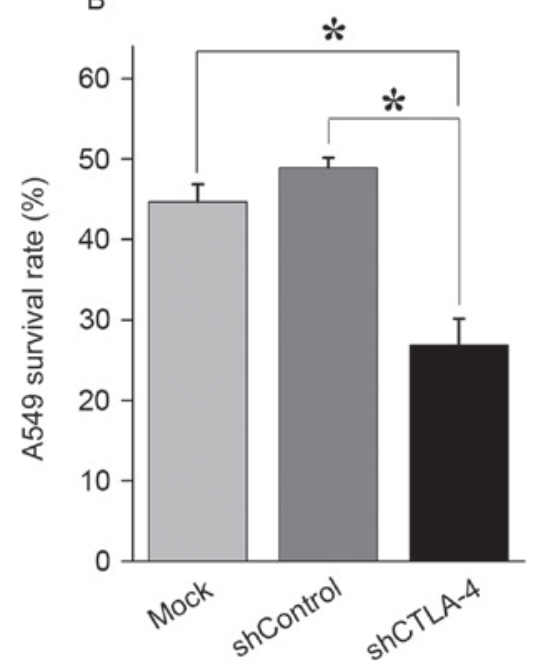

$\mathrm{C}$
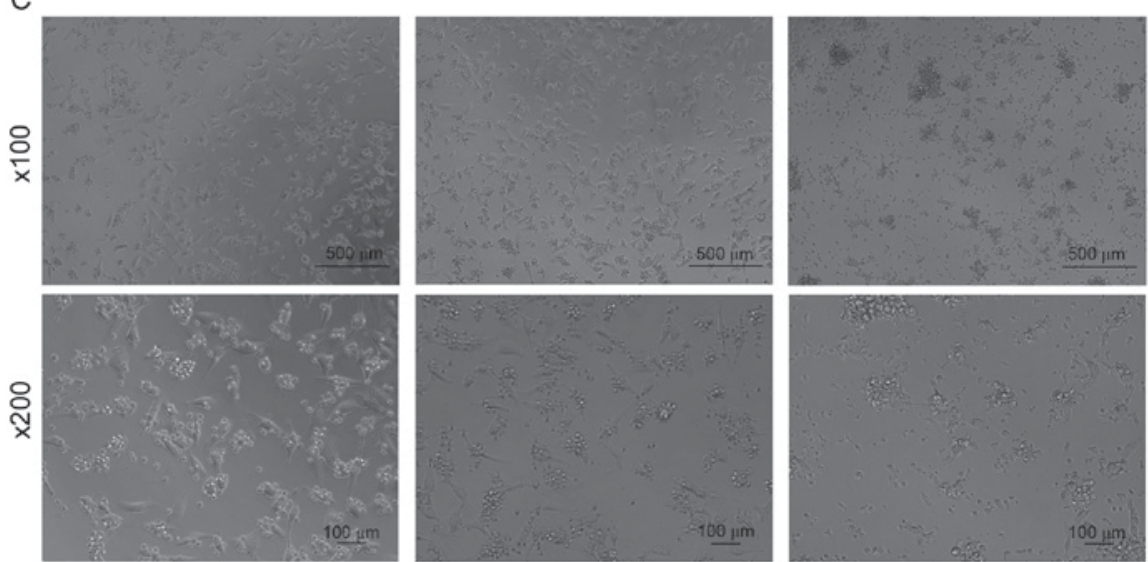

shControl

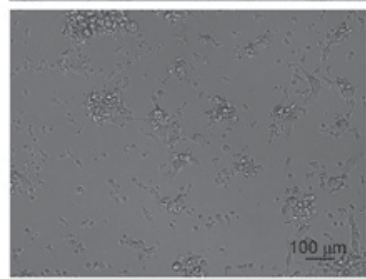

ShCTLA-4

Figure 4. The inhibition of CTLA-4 expression increased cytotoxicity of CIK cells against A549 cells. (A) Determined by CytoTox 96 Non-Radioactive Cytotoxicity Assay, compared with CIK cells mock transduced or transduced with shControl lentiviral particles, CIK cells transduced with shCTLA-4 lentiviral particles exhibited significantly higher cytotoxicity against A549 cells. $\mathrm{P}<0.001$. (B) A549 cells were co-cultured with CIK cells for $24 \mathrm{~h}$ before cell counting. Compared with the number of viable A549 cells co-cultured with CIK cells mock transduced or transduced with shControl lentiviral particles, the number of viable A549 cells co-cultured with CIK cells transduced with shCTLA-4 lentiviral particles were significantly lower. "P<0.01. (C) Representative figures of A549 cells co-cultured with CIK cells mock transduced (left panel), transduced with shControl lentiviral particles (middle panel), and transduced with shCTLA-4 lentiviral particles (right panel) for $24 \mathrm{~h}$. Each experiment was performed three times. The data were plotted as the mean \pm standard error of the mean. CTLA-4, cytotoxic T lymphocyte-associated antigen 4; CIK, cytokine-induced killer; PMBC, peripheral blood mononuclear cells; qPCR, quantitative polymerase chain reaction; sh, short hairpin.

the regulation of CTLA-4: CD28/CD80 axis. It was reported that CD80 serves a pivotal role in immune cell-mediated cytotoxicity. Chambers et al (20) indicates that NK cell-mediated cytotoxicity is a result of interactions between triggering signals and the inhibitory receptors and ligands, during which CD80 isthe triggering signal for tumor cell lysis.

In the present study, we demonstrated that the expression of CTLA-4 on CIK cells is upregulated compared with that on the corresponding PBMCs. This is concordant with the results of previous reports, in that the responsiveness of IL-2 led to the induction of CLTA-4, particularly for the IL-2-expanded immune effector cells $(21,22)$. It was hypothesized that PBMCs, when induced with a CIK cell cytokine cocktail, are activated and this trigged the activation of inhibitory signal to balance the immune response of CIK cells represented by the concurrent upregulation of CTLA-4 and CD28 $(23,24)$.

It was further demonstrated that the surpression of CTLA-4 expression by lentiviral vector-mediated RNA interference
(RNAi) on CIK cells promoted proliferation and the killing efficiency of CIK cells, which confirmed CTLA-4's inhibitory effect on CIK cells. Since regulatory $\mathrm{T}$ cells $\left(\mathrm{T}_{\text {regs }}\right)$ are crucial for the maintenance of immune tolerance, especially for autoimmunity and the tumor microenvironment (25-27), it was hypothesized that the effect of inhibiting CTLA-4 expression on CIK cells may alter the balance of $\mathrm{T}_{\text {regs }} . \mathrm{T}_{\text {regs }}$ express a high level of CD25 (the IL-2 receptor $\alpha$ chain) and compete with other effector cells to bind IL-2, resulting in cytokine-mediated immune suppression $(6,28)$. Furthermore, it has been reported that the interaction of CTLA-4 on $\mathrm{T}_{\text {regs }}$ with CD80 and CD86 on APCs can block and even downregulates the expression of CD80 and CD86 (29). It was also reported that blocking CTLA-4 has an impact on the balance of $\mathrm{T}_{\text {regs }}$ and downregulates $\mathrm{T}_{\text {regs }}$-mediated suppression, but the mechanisms are still unclear $(23,30)$.

Although the results have revealed the immune suppression of CIK cells mediated by CTLA-4, they were unable to 
determine the exact mechanisms by which CTLA-4 regulates the proliferation and activation of CIK cells, which is the distinct limitation of this study.

To conclude, the present study demonstrated that CTLA-4 expression increased when PBMCs were induced into CIK cells by cytokine cocktail, RNAi mediated by lentiviral particles efficiently inhibited the expression of CTLA-4 on CIK cells, suppression of CTLA-4 expression significantly promoted the proliferation of CIK cells in vitro and enhanced the cytotoxicity of CIK cells against lung cancer cells. These findings indicate that the blockade of CTLA-4 signaling has potential therapeutic significance for CIK cell therapy and further clinical study is warranted to verify these results.

\section{References}

1. Rosenberg SA: Progress in human tumour immunology and immunotherapy. Nature 411: 380-384, 2001.

2. Bour-Jordan H and Blueston JA: CD28 function: A balance of costimulatory and regulatory signals. J Clin Immunol 22: 1-7, 2002.

3. van Gool SW, Barcy S, Devos S, Vandenberghe P, Ceuppens JL, Thielemans K and de Boer M: CD80 (B7-1) and CD86 (B7-2): Potential targets for immunotherapy. Res Immunol 146: 183-196, 1995.

4. Slavik JM, Hutchcroft JE and Bierer BE: CD28/CTLA-4 and CD80/CD86 families: Signaling and function. Immunol Res 19: $1-24,1999$.

5. Nakajima A and Azuma M: Costimulatory molecules in autoimmunity: Role of CD28/CTLA4-CD80/CD86. Nihon Rinsho 55: 1419-1424, 1997 (In Japanese).

6. Krummel MF and Allison JP: CTLA-4 engagement inhibits IL-2 accumulation and cell cycle progression upon activation of resting T cells. J Exp Med 183: 2533-2540, 1996.

7. Soskic B, Qureshi OS, Hou T and Sansom DM: A transendocytosis perspective on the CD28/CTLA-4 pathway. Adv Immunol 124: 95-136, 2014.

8. Darcy PK, Neeson P, Yong CS and Kershaw MH: Manipulating immune cells for adoptive immunotherapy of cancer. Curr Opin Immunol 27: 46-52, 2014

9. Margolin KA, Negrin RS, Wong KK, Chatterjee S, Wright $\mathrm{C}$ and Forman SJ: Cellular immunotherapy and autologous transplantation for hematologic malignancy. Immunol Rev 157: 231-240, 1997.

10. Schmeel LC, Schmeel FC, Coch C and Schmidt-Wolf IG: Cytokine-induced killer (CIK) cells in cancer immunotherapy: Report of the international registry on CIK cells (IRCC). J Cancer Res Clin Oncol 141: 839-849, 2015.

11. Schmidt-Wolf IG, Negrin RS, Kiem HP, Blume KG and Weissman IL: Use of a SCID mouse/human lymphoma model to evaluate cytokine-induced killer cells with potent antitumor cell activity. J Exp Med 174: 139-149, 1991.

12. Mesiano G, Todorovic M, Gammaitoni L, Leuci V, Giraudo Diego L, Carnevale-Schianca F, Fagioli F, Piacibello W, Aglietta M and Sangiolo D: Cytokine-induced killer (CIK) cells as feasible and effective adoptive immunotherapy for the treatment of solid tumors. Expert Opin Biol Ther 12: 673-684, 2012.

13. Chung MJ, Park JY, Bang S, Park SW and Song SY: Phase II clinical trial of ex vivo-expanded cytokine-induced killer cells therapy in advanced pancreatic cancer. Cancer Immunol Immunother 63: 939-946, 2014.
14. Liang XF, Ma DC, Ding ZY, Liu ZZ, Guo F, Liu L, Yu HY, Han YL and Xie XD: Autologous cytokine-induced killer cells therapy on the quality of life of patients with breast cancer after adjuvant chemotherapy: A prospective study. Zhonghua Zhong Liu Za Zhi 35: 764-768, 2013.

15. Yu X, Zhao H, Liu L, Cao S, Ren B, Zhang N, An X, Yu J, $\mathrm{Li} \mathrm{H}$ and Ren X: A randomized phase II study of autologous cytokine-induced killer cells in treatment of hepatocellular carcinoma. J Clin Immunol 34: 194-203, 2014.

16. Zhong R, Han B and Zhong H: A prospective study of the efficacy of a combination of autologous dendritic cells, cytokine-induced killer cells, and chemotherapy in advanced non-small cell lung cancer patients. Tumour Biol 35: 987-994, 2014.

17. Robert L, Harview C, Emerson R, Wang X, Mok S, Homet B, Comin-Anduix B, Koya RC, Robins H, Tumeh PC and Ribas A: Distinct immunological mechanisms of CTLA-4 and PD-1 blockade revealed by analyzing TCR usage in blood lymphocytes. Oncoimmunology 3: e29244, 2014.

18. Qureshi OS, Zheng Y, Nakamura K, Attridge K, Manzotti C, Schmidt EM, Baker J, Jeffery LE, Kaur S, Briggs Z, et al: Trans-endocytosis of CD80 and CD86: A molecular basis for the cell-extrinsic function of CTLA-4. Science 332: 600-603, 2011.

19. Catalfamo M, Tai X, Karpova T, McNally J and Henkart PA: TcR-induced regulated secretion leads to surface expression of CTLA-4 in CD4 ${ }^{+} \mathrm{CD} 25^{+} \mathrm{T}$ cells. Immunology 125: 70-79, 2008.

20. Chambers BJ, Salcedo M and Ljunggren HG: Triggering of natural killer cells by the costimulatory molecule CD80 (B7-1). Immunity 5: 311-317, 1996.

21. Geldhof AB, Moser M, Lespagnard L, Thielemans K and De Baetselier P: Interleukin-12-activated natural killer cells recognize B7 costimulatory molecules on tumor cells and autologous dendritic cells. Blood 91: 196-206, 1998.

22. Stojanovic A, Fiegler N, Brunner-Weinzierl M and Cerwenka A: CTLA-4 is expressed by activated mouse NK cells and inhibits NK Cell IFN- $\gamma$ production in response to mature dendritic cells. J Immunol 192: 4184-4191, 2014.

23. Quezada SA, Peggs KS, Curran MA and Allison JP: CTLA4 blockade and GM-CSF combination immunotherapy alters the intratumor balance of effector and regulatory $\mathrm{T}$ cells. J Clin Invest 116: 1935-1945, 2006.

24. Egen JG, Kuhns MS and Allison JP: CTLA-4: New insights into its biological function and use in tumor immunotherapy. Nat Immunol 3: 611-618, 2002.

25. Yamaguchi $\mathrm{T}$ and Sakaguchi $\mathrm{S}$ : Regulatory $\mathrm{T}$ cells in immune surveillance and treatment of cancer. Semin Cancer Biol 16 115-123, 2006.

26. Sakaguchi S: Naturally arising Foxp3-expressing CD $25^{+} \mathrm{CD} 4^{+}$ regulatory $\mathrm{T}$ cells in immunological tolerance to self and non-self. Nat Immunol 6: 345-352, 2005.

27. Friedline RH, Brown DS, Nguyen H, Kornfeld H, Lee J, Zhang Y, Appleby M, Der SD, Kang J and Chambers CA: CD4 ${ }^{+}$regulatory $\mathrm{T}$ cells require CTLA- 4 for the maintenance of systemic tolerance. J Exp Med 206: 421-434, 2009.

28. Josefowicz SZ, Lu LF and Rudensky AY: Regulatory T cells: Mechanisms of differentiation and function. Annu Rev Immunol 30: 531-564, 2012.

29. Onishi Y, Fehervari Z, Yamaguchi T and Sakaguchi S: Foxp3 ${ }^{+}$ natural regulatory $\mathrm{T}$ cells preferentially form aggregates on dendritic cells in vitro and actively inhibit their maturation. Proc Natl Acad Sci USA 105: 10113-10118, 2008.

30. Read S, Greenwald R, Izcue A, Robinson N, Mandelbrot D, Francisco L, Sharpe AH and Powrie F: Blockade of CTLA-4 on $\mathrm{CD} 4{ }^{+} \mathrm{CD} 25^{+}$regulatory $\mathrm{T}$ cells abrogates their function in vivo. J Immunol 177: 4376-4383, 2006.

This work is licensed under a Creative Commons Attribution-NonCommercial-NoDerivatives 4.0 International (CC BY-NC-ND 4.0) License. 